\title{
Noise characterization of a single parameter quantized charge pump
}

\author{
F. Hohls*, N. Maire*, B. Kaestner ${ }^{\dagger}$, K. Pierz ${ }^{\dagger}$, H. W. Schumacher ${ }^{\dagger}$ and \\ R. J. Haug* \\ ${ }^{*}$ Institut für Festkörperphysik, Leibniz Universität Hannover, Appelstr. 2, 30167 Hannover, \\ Germany \\ ${ }^{\dagger}$ Physikalisch-Technische Bundesanstalt, Bundesallee 100, 38116 Braunschweig, Germany
}

\begin{abstract}
The shot noise of a single parameter quantized charge pump is studied. The pumped current can be varied using a control gate. Quantized current plateaus $I=n e f_{p}$ with $f_{p}$ the pumping frequency and $e$ the electron charge are observed. The shot noise is minimal for each current plateau and maximal in between. Interestingly the first expected quantized current plateau at $n=1$ is missing for certain control gate voltages. We use the measured shot noise to extract the probabilities for pumping none, one or two electrons at the position of the missing step. These probabilities can be used to characterize the dynamics of the non-adiabatic pumping process.
\end{abstract}

Keywords: Single electron effects, quantized charge pump, shot noise PACS: 72.70.+m, 73.23.Hk, 73.23.-b, 73.63.Kv

A quantized charge pump is a device that delivers a well controlled number $n$ of electrons in each cycle of the driving frequency $f_{p}$. Doing this with sufficiently high frequency and reliability and thus creating a current $I=n e f_{p}$ would allow to realize a quantum standard for the ampere $[1,2,3]$. However, most approaches to realize quantized pumping $[4,5,6,7,8]$ either lack the necessary accuracy or do not obtain sufficiently high currents. Recently a new promising realization was demonstrated [9]: It consists of a semiconductor quantum wire crossed by three metal gates which can be used to create potential barriers within the wire. For pumping, two of these gates were driven by phase locked high frequency signals in this initial demonstration. Recently Kaestner et al. have shown experimentally that driving a single gate is sufficient to achieve a quantized pumping current at very high frequency $[10,11,12,13]$. A theoretical analysis of this non-adiabatic charge pumping promises that an optimized device could reach metrological accuracy [10].

The working principle of this new non-adiabatic charge pump is the following: The potential barriers generated by two energized Schottky gates form a quantum dot within the wire. The additional ac-voltage applied to one of the gates first reduces one of the barriers and at the same time the dot level. The latter is reduced to below the Fermi energy, allowing the loading of electrons from the source onto the quantum dot. Then this barrier is raised, forming an isolated quantum dot. During this process some electrons are expelled dynamically from the dot and only a number $n$ of electrons which is determined by details of the system and the gate voltages remains within the dot [14]. Applying an even higher voltage expels the electrons into the drain and finishes the pumping cycle. The number of electrons $n$ pumped in each cycle is determined by a fine balance of

CP1129, Noise and Fluctuations, 20 International Conference (ICNF 2009)

edited by M. Macueci and G. Basso

2009 American Institute of Physics 978-0-7354-0665-0/09/\$25.00 
the dynamic confinement potential and the Coulomb energy and can be varied by the applied dc and ac voltages.

A perfect quantized charge pump would be completely deterministic, pumping exactly $n$ electrons in every cycle. As a result one expects vanishing low frequency noise power. For a non-ideal pump with non-zero probabilities of differing electron numbers per cycle a non-zero low frequency current noise $S_{I}(f)=S_{I}^{0}\left(f \ll f_{p}\right)$ is expected $[15,16]$. Thus we can use a shot noise measurement to characterize the charge pump.

In this paper we present such noise measurements of a single parameter quantized charge pump. The pump is formed by three $100 \mathrm{~nm}$-wide metallic finger gates sitting on top of a semiconductor wire etched in a $n$-type AlGaAs heterostructure (Fig. 1c). Voltages are applied to two finger gates to form potential barriers underneath. One of the finger gates is additionally driven by a sinusoidal voltage at a frequency of several hundred $\mathrm{MHz}$ (Fig. 1b). The measurements are performed in a home built ${ }^{3} \mathrm{He}$ cryostat at a temperature of $0.4 \mathrm{~K}$ using a special low capacitance probe and a very low noise current amplifier $[11,17,18]$.

When varying the dc voltage applied to the finger gates and measuring the dc current we observe plateaus with quantized current values $I=n e f_{p}$ indicating quantized charge pumping (Fig. 1a). In addition to the dc current the noise power is measured for frequencies $f \leq 15 \mathrm{kHz}$. Exemplary spectra are shown in Fig. 1d, one taken on a current plateau $(I=2 e f)$, the other one at a non-quantized current $(I=1.7 e f)$. Indeed the shot noise is strongly suppressed for the quantized current value $(I=2 e I)$ and is nonzero for the non-quantized current value (1.7ef) in agreement with our recent study in Ref. [11].

We will now focus on the measured shot noise for non-quantized current values. We examine the dependence of the current and noise as function of the voltage $V_{2}$ applied to the static gate (in Ref. [11] $V_{1}$ was varied). Fig. 2a shows the normalized current as function of $V_{2}$. Plateaus appear at $I=2 e f_{p}, 3 e f_{p}$ and $4 e f_{p}$ while the $I=e f_{p}$ plateau is missing. The measured shot noise is shown in Fig. 2b. It vanishes within the measurement resolution of $\Delta S \leq 10^{-30} \mathrm{~A}^{2} / \mathrm{Hz}$ at the $I=2 e f_{p}$ plateau and shows minima at $3 e f_{p}$ and $4 e f_{p}$, but interestingly the noise has a maximum at $I=e f_{p}$, marked by the dashed circle.

In Ref. [11] it was found that the non-zero noise power for current values $(n-1) e f_{p}<$ $I<n e f_{p}$ is well modelled taking into account only two different possible pumping processes, i.e. having $n-1$ or $n$ electrons per cycle. Using this assumption one can calculate the expected noise power from the current. The result is displayed as black line in Fig. 2b. A good agreement with the measured noise is observed for $V<-110 \mathrm{mV}$ and for $V>-100 \mathrm{mV}$. But in between these voltages near the maximum in the shot noise at the missing $I=e f_{p}$ step it is clearly not sufficient to consider only two processes.

When regarding three different possible numbers of pumped electrons with probabilities $p_{n-1}, p_{n}$ and $p_{n+1}$ with $p_{n-1}+p_{n}+p_{n+1}=1$ the current and the shot noise are given by $[15,16]$

$$
I=\left(n+p_{n+1}-p_{n-1}\right) e f_{p} \quad \text { and } \quad S=2\left[p_{n-1}+p_{n+1}-\left(p_{n-1}-p_{n+1}\right)^{2}\right] e^{2} f_{p} .
$$

We can use these relations to determine the probabilities $p_{0}, p_{1}$ and $p_{2}$ relevant around the missing current plateau in between $I=0$ and $I=2 e f_{p}$. Fig. $2 \mathrm{~d}$ shows the result. Starting from nearly zero current $\left(p_{0} \approx 1\right)$ initially $p_{1}$ rises and $p_{0}$ drops according to 

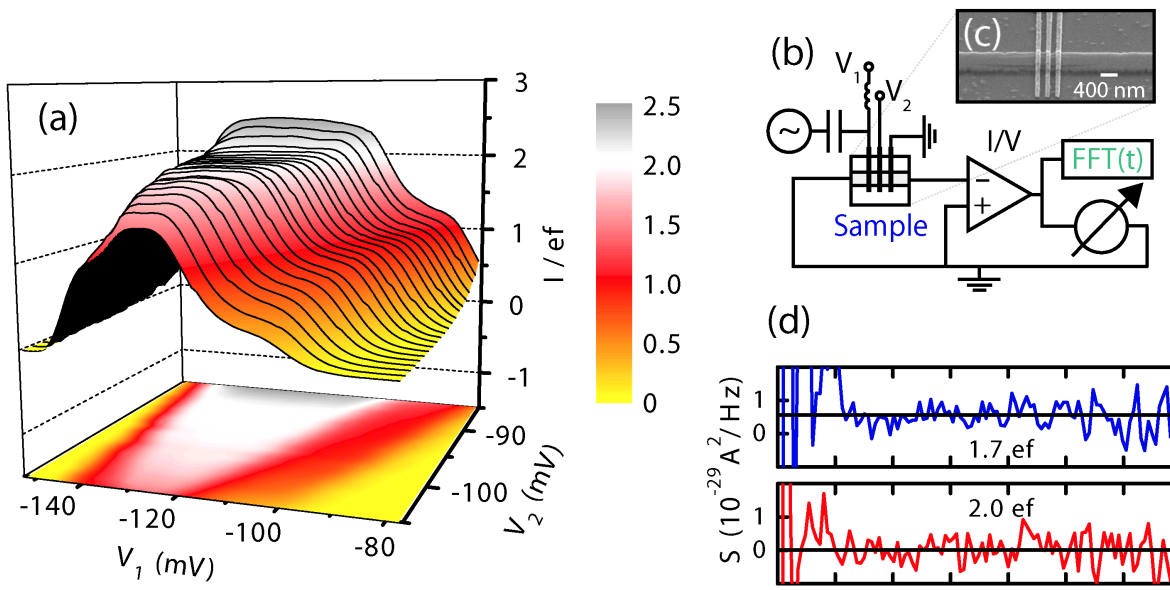

(d)

0.5

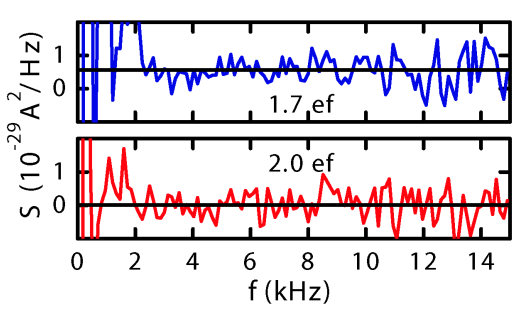

FIGURE 1. a) Normalized pumped current at a frequency of $f=400 \mathrm{MHz}$ as function of the two control gate voltages. b) Schematic of the measurement setup. c) SEM micrograph of the device shows the etched quantum wire that is crossed by three metallic gates. d) Selected noise power spectra for the voltage settings marked by the blue and red solid symbols in Fig. 2a. The horizontal line shows the averaged level in the range $f=5-15 \mathrm{kHz}$.
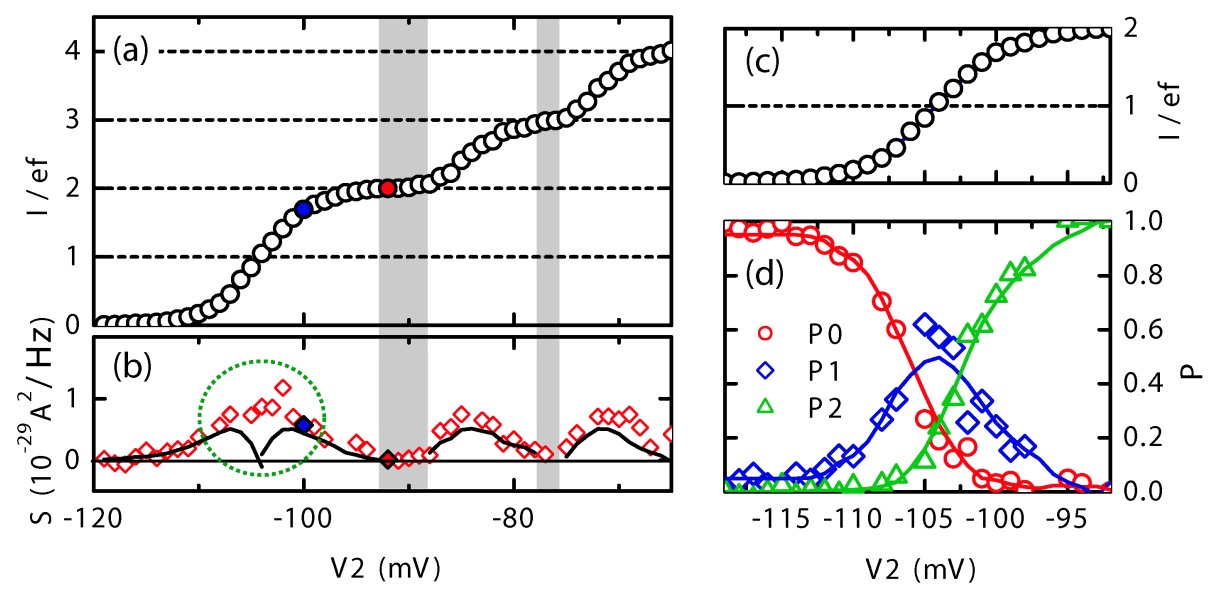

FIGURE 2. a) Normalized current as function of $V_{2}$ for $f=400 \mathrm{MHz}$ and $V_{1}=-130 \mathrm{mV}$. b) Measured shot noise power (Symbols, noise power average over $f=5-15 \mathrm{kHz}$ ). The line shows the expectation for only two non-zero pumping probabilities. $\mathrm{c}+\mathrm{d}$ ) Current and calculated pumping probabilities throughout the initial current step. 
$p_{0}+p_{1}=1$. For a $I=e f_{p}$ plateau we would expect that $p_{1}$ reaches one while $p_{0}$ goes to zero. But instead $p_{2}$ starts to rise already for $p_{0} \approx 0.5$ and $p_{1}$ reaches only a maximum value of $p_{1, \max } \approx 0.5$ with $p_{0} \approx p_{2} \approx 0.25$ at $I \approx e f_{p}$. This reveals that the dynamics of the system in this regime does not favour a single probability, leading to the observed "missing step" and enhanced noise. This behaviour deviates from the cascade model of the dot loading described in Ref. [14]. Thus we need a new model for this regime of the charge pump which will be tested with our data.

In conclusion we have measured the current and the shot noise of a single parameter quantized charge pump as function of its control gate voltages. We have shown that the noise measurements can be used to characterize the probabilities of different electron numbers transferred in one pumping cycle. We examined the occurrence of a "missing step" which reveals an interesting regime of the dynamics of the quantum dot formed in the pump.

The authors would like to thank G. Hein, Th. Weimann, and H. Marx for support of clean room processing and sample growth. Funding was provided by the BMBF via project nanoQUIT.

\section{REFERENCES}

1. D. V. Averin, and K. K. Likharev, J. Low Temp. Phys. 62, 345-373 (1986).

2. K. Flensberg, A. A. Odintsov, F. Liefrink, and P. Teunissen, Int. J. Mod. Phys. B 13, 2651 (1999).

3. I. M. Mills, P. J. Mohr, T. J. Quinn, B. N. Taylor, and E. R. Williams, Metrologia 43, 227-246 (2006).

4. L. J. Geerligs, V. F. Anderegg, P. A. M. Holweg, J. E. Mooij, H. Pothier, D. Esteve, C. Urbina, and M. H. Devoret, Phys. Rev. Lett. 64, 2691-2694 (1990).

5. L. P. Kouwenhoven, A. T. Johnson, N. C. van der Vaart, C. J. P. M. Harmans, and C. T. Foxon, Phys. Rev. Lett. 67, 1626-1629 (1991).

6. H. Pothier, P. Lafarge, C. Urbina, D. Esteve, and M. H. Devoret, Europhys. Lett. 17, 249-254 (1992), ISSN 0295-5075.

7. M. W. Keller, J. M. Martinis, N. M. Zimmerman, and A. H. Steinbach, Appl. Phys. Lett. 69, 18041806 (1996).

8. J. M. Shilton, V. I. Talyanskii, M. Pepper, D. A. Ritchie, J. E. F. Frost, C. J. B. Ford, C. G. Smith, and G. A. C. Jones, J. Phys.: Condens. Matter 8, L531-L539 (1996), ISSN 0953-8984.

9. M. D. Blumenthal, B. Kaestner, L. Li, S. Giblin, T. J. B. M. Janssen, M. Pepper, D. Anderson, G. Jones, and D. A. Ritchie, Nat Phys 3, 343 (2007), ISSN 1745-2481.

10. B. Kaestner, V. Kashcheyevs, S. Amakawa, M. D. Blumenthal, L. Li, T. J. B. M. Janssen, G. Hein, K. Pierz, T. Weimann, U. Siegner, and H. W. Schumacher, Phys. Rev. B 77, 153301 (2008).

11. N. Maire, F. Hohls, B. Kaestner, K. Pierz, H. W. Schumacher, and R. J. Haug, Appl. Phys. Lett. 92 , $082112(2008)$.

12. B. Kaestner, V. Kashcheyevs, G. Hein, K. Pierz, U. Siegner, and H. W. Schumacher, Appl. Phys. Lett. 92, 192106 (2008).

13. B. Kaestner, C. Leicht, V. Kashcheyevs, K. Pierz, U. Siegner, and H. W. Schumacher, Appl. Phys. Lett. 94, 012106 (2009).

14. V. Kashcheyevs, and B. Kaestner, Universal decay cascade model for dynamical quantum dot initialization, arXiv:0901.4102v1 (2009).

15. Y. M. Galperin, O. Entin-Wohlman, and Y. Levinson, Phys. Rev. B 63, 153309 (2001).

16. A. M. Robinson, and V. I. Talyanskii, Phys. Rev. Lett. 95, 247202 (2005).

17. A. Nauen, I. Hapke-Wurst, F. Hohls, U. Zeitler, R. J. Haug, and K. Pierz, Phys. Rev. B 66, 161303R (2002).

18. N. Maire, F. Hohls, T. Luedtke, K. Pierz, and R. J. Haug, Phys. Rev. B 75, 233304 (2007). 
Copyright of AIP Conference Proceedings is the property of American Institute of Physics and its content may not be copied or emailed to multiple sites or posted to a listserv without the copyright holder's express written permission. However, users may print, download, or email articles for individual use. 